\title{
Crowned dens syndrome: Caution about asymptomatic calcification
}

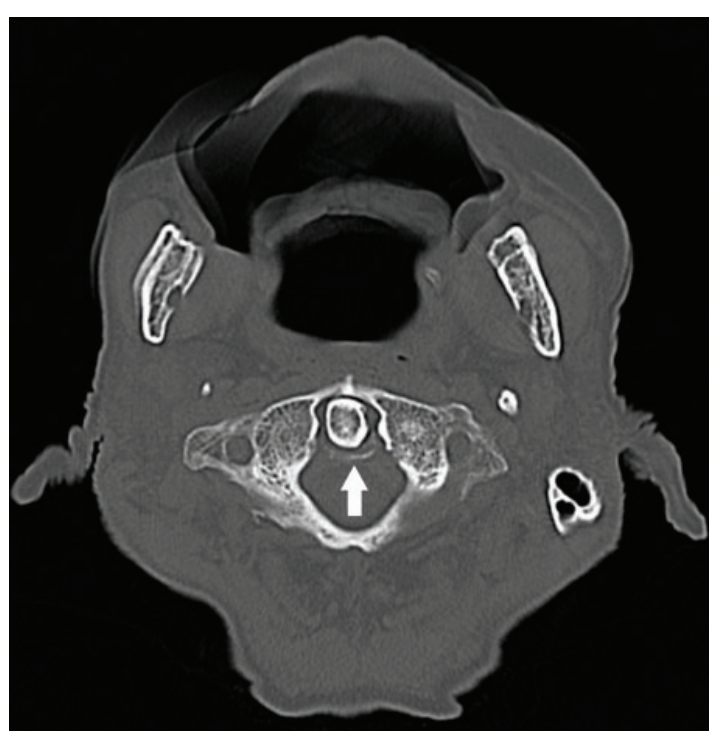

Figure 1. Noncontrast computed tomography of the head and neck showed calcification around the dens (arrow).

\section{of the central} nervous system needs prompt intervention
A N 88-YEAR-Old JAPANESE MAN in a psychiatric hospital because of progressive $\mathrm{Al}$ zheimer disease developed fever, headache, and posterior neck pain. The symptoms persisted despite antibiotic therapy with $500 \mathrm{mg} /$ day of oral levofloxacin and $500 \mathrm{mg} /$ day of intravenous meropenem. He was transported to our emergency department 42 days after the onset of symptoms.

See related article, page 206

On chest auscultation, the lungs were clear bilaterally and heart sounds were normal. There was no nuchal rigidity, red eye, muscular pain, arthralgia, rash, or abdominal pain. Laboratory tests revealed a white blood cell count of $3.43 \times 10^{9} / \mathrm{L}$ (reference range 3.59-9.64) and a C-reactive protein level of $0.95 \mathrm{mg} / \mathrm{dL}(0-0.30)$.

Computed tomography of the head and neck without contrast enhancement showed calcium deposition around the dens (Figure 1). Cerebrospinal fluid obtained by lumbar puncture was colorless and transparent and showed no red blood cells or white blood cells.

Based on the persistence of headache and neck pain despite antibiotic therapy and on calcification around the dens, a tentative diagnosis of crowned dens syndrome was made. The patient was prescribed a nonsteroidal anti-inflammatory drug (loxoprofen $60 \mathrm{mg}$ ), to be used as needed.

Bacterial culture of the cerebrospinal fluid came back 2 days later positive for Bacillus cereus. Therefore, the patient was diagnosed with bacterial meningitis, partially treated with previously administered antibiotics. He was quickly admitted to our hospital and started on intravenous vancomycin $750 \mathrm{mg}$ every 24 hours and clindamycin $600 \mathrm{mg}$ every 8 hours. His fever, headache, and cervical pain diminished gradually, and he was discharged to home on day 57.

\section{IS IT INFECTION OR CROWNED DENS?}

Crowned dens syndrome is characterized by acute-onset fever, head and cervical pain, and radiographic findings of calcium deposition around the dens. ${ }^{1}$ The calcification in our patient was thin, with no adjacent soft-tissue 
swelling. Advances in computed tomography enable the detection of slight calcifications ${ }^{2}$; slight calcifications may be normal and should not raise unnecessary suspicion for crowned dens syndrome.

This patient's case conveys the important message that calcium deposition in the atlantoaxial joint is not always symptomatic. It remained unclear whether the patient developed crowned dens syndrome with infection or just the infection.

Although crowned dens syndrome is relatively benign, infection of the central nervous system is a critical condition that needs prompt intervention. Physicians should not rule out infectious disease based only on a finding of calcium deposition.

\section{HEEDING THE CLUES}

Asymptomatic atlantoaxial calcium deposition is reportedly common in the aged population. ${ }^{3}$ In the present case, the prolonged period of fever was inconsistent with crowned

\section{REFERENCES}

1. Bouvet JP, le Parc JM, Michalski B, Benlahrache C, Auquier L. Acute neck pain due to calcifications surrounding the odontoid process: the crowned dens syndrome. Arthritis Rheum 1985; 28(12):1417-1420. doi:10.1002/art.1780281215

2. Miksanek J, Rosenthal AK. Imaging of calcium pyrophosphate deposition disease. Curr Rheumatol Rep 2015; 17(3):20. doi:10.1007/s11926-015-0496-1 dens syndrome, which usually develops acutely and subsides within a few days. In addition, inappropriate antibiotic use might lead to partial treatment of meningitis, pseudonegative cerebrospinal fluid findings, and, in this case, delayed diagnosis.

In summary, physicians should be aware that the combination of fever, headache, cervical pain, and calcium deposition around the dens does not always indicate crowned dens syndrome. Asymptomatic calcification is not rare, and physicians should not hesitate to perform lumbar puncture and other diagnostic tests to rule out life-threatening diseases such as bacterial meningitis.

Acknowledgments: I thank Dr. Hotaka Hara for engaging in useful discussion and providing clinical instruction. I also thank Kelly Zammit, BVSc, from Edanz Group (https://enauthor-services.edanzgroup.com/ac) for editing a draft of this manuscript.

\section{DISCLOSURES}

The author reports no relevant financial relationships which, in the context of the contributions, could be perceived as a potential conflict of interest.

3. Chang EY, Lim WY, Wolfson T, et al. Frequency of atlantoaxial calcium pyrophosphate dihydrate deposition at CT. Radiology 2013; 269(2):519-524. doi:10.1148/radiol.13130125

Address: Junki Mizumoto, MD, Department of Medical Education Studies, International Research Center for Medical Education, Graduate School of Medicine, The University of Tokyo, 7-3-1 Hongou, Bunkyo-ku, Tokyo, 113-0033 Japan; jnk_mizu@yahoo.co.jp 